\title{
Drug-associated histiocytoid Sweet's syndrome: a true neutrophilic maturation arrest variant
}

Histiocytoid Sweet's syndrome is a recently described entity which has clinical features identical to typical Sweet's syndrome but is distinguished by a dermal cellular infiltrate composed not of mature neutrophils but of immature granulocytes. Herein, we report a case of bone marrow granulocytic maturation arrest and a histological histiocytoid Sweet's-like reaction pattern following trimethoprimsulfamethoxazole therapy.

Wu AJ, Rodgers T, Fullen DR. Drug-associated histiocytoid Sweet's syndrome: a true neutrophilic maturation arrest variant.

J Cutan Pathol 2008; 35: 220-224. (C) Blackwell Munksgaard 2007.

\section{Angela J. Wu' ${ }^{1}$, Timothy Rodgers ${ }^{2}$ and Douglas $R$. Fullen $^{1,2}$}

${ }^{1}$ Department of Pathology and

2Department of Dermatology, University of

Michigan Medical Center, Ann Arbor, MI, USA

\section{Dr Douglas R. Fullen, University of Michigan}

Medical Center, M3261 MSI 0602, 1301 Catherine

Road, Ann Arbor, MI 48109-0602, USA

Tel: +17347644460

Fax: +1 7347644690

e-mail: dfullen@med.umich.edu

Accepted for publication March 12, 2007
Acute febrile neutrophilic dermatosis or Sweet's syndrome, is a reactive condition of unknown etiology characterized by an abrupt onset of tender, erythematous or violaceous sharply marginated plaques and nodules, occasionally associated with pseudovesiculation or pustules. Lesions are asymmetrically distributed over the face, neck and limbs. Associated secondary features include clinical symptoms such as fever and characteristic laboratory values including neutrophilic leukocytosis, elevated erythrocyte sedimentation rate and positive $\mathrm{C}$-reactive protein. The classic histomorphology of Sweet's syndrome is one of a dichotomous reaction pattern comprising striking interstitial neutrophilia accompanied by a mononuclear cell vascular reaction typically without mural and or luminal fibrin deposition. The mononuclear cells may be of monocytic or lymphoid derivation. An underlying systemic disorder, most often a viral or streptococcal infection or inflammatory condition such as inflammatory bowel disease, is found in approximately $50 \%$ of cases. Approximately 10$20 \%$ of cases are associated with hematologic or solid malignancies. ${ }^{1,2}$

Recently, Requena et al. described a series of 41 patients who presented with typical cutaneous lesions of Sweet's syndrome. Histologically, these lesions had the architectural features of Sweet's syndrome, but the cellular infiltrate was composed predominantly of immature myeloid precursors without the morphologic features of either blasts or segmented neutrophils. Requena et al. held that the granulocytic nature of the cells was supported by the phenotypic profile, namely, one comprising CD68, lysozyme and myeloperoxidase (MPO) positivity. However, it should be emphasized that while MPO activity is most pronounced in immature granulocytes, it is discernible in monocytes as well. A truly confirmatory marker of granulocytic derivation, namely that of chloroacetate esterase, along with negativity for a monocyte-specific marker, such as a non-specific esterase stain using alpha naphthyl acetate and alpha naphthyl butyrate was not performed. Because of the morphologic resemblance of these immature myeloid precursors to histiocytes, this dermatosis was coined histiocytoid Sweet's syndrome. ${ }^{2}$

In this case report, we present an unusual case of histiocytoid Sweet's-like reaction and granulocytic maturation arrest in a 44-year-old woman following a course of trimethoprim-sulfamethoxazole (TMPSFX) therapy for treatment of sinusitis. 


\section{Clinical presentation}

This patient was a 44-year-old previously healthy woman who presented to her primary care physician with complaints of sinusitis; a 10-day course of TMP-SFX was prescribed. Five days into the antibiotic course, the patient developed myalgia and fever for 2 days. These symptoms improved, but approximately 1 week later, she noticed an erythematous nodule over her left ribcage and again developed fever and myalgia. Over the next few days, this nodule grew, developed vesicles and became painful. Her primary care physician suspected a spider bite and prescribed a dose of cephalexin and ceftriaxone. On routine laboratory tests, the patient was found to have a low white blood cell (WBC) count; she was informed of these results and directed to go to the Emergency Department.

\section{Physical examination and laboratory values}

On presentation, the patient had a fever of $101.3^{\circ} \mathrm{F}$, blood pressure $118 / 78 \mathrm{mmHg}$, heart rate $106 \mathrm{bpm}$ and respiratory rate $20 \mathrm{rpm}$. Physical examination showed a 1.5-cm hemorrhagic nodule on the left flank with pseudovesiculation and surrounding erythema measuring approximately $10 \mathrm{~cm}$ in diameter (Fig. 1). This lesion was somewhat tender to palpation. No other skin or oral lesions were present. Laboratory values were as follows: WBC count $1,200 \mathrm{~mm}^{3}$, hemoglobin $12.3 \mathrm{~g} / \mathrm{dl}$, hematocrit $35.9 \%$, platelets $359,000 \mathrm{~mm}^{3}$; absolute neutrophil count (ANC) $0 \mathrm{~mm}^{3}$, absolute lymphocyte count $1,000 \mathrm{~mm}^{3}$, absolute monocyte count $300 \mathrm{~mm}^{3}$. A peripheral blood smear review showed no blasts, no neutrophils and rare band neutrophils. A dermatology and hematology consult were obtained. The

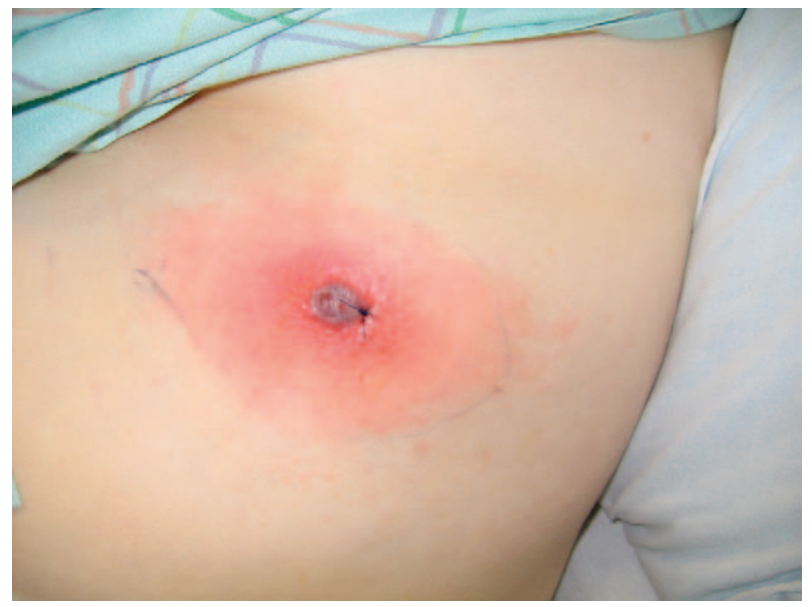

Fig. 1. The patient had a 1.5-cm hemorrhagic nodule over her left flank with surrounding pseudovesiculation and erythema measuring approximately $10 \mathrm{~cm}$ in diameter.

\section{Drug-associated histiocytoid Sweet's syndrome}

patient subsequently underwent skin and bone marrow biopsies. The differential diagnosis included an atypical presentation of Sweet's syndrome secondary to TMP-SMX, leukemia cutis, arthropod bite reaction, atypical pyogenic granuloma and an infectious process.

\section{Histopathology}

A biopsy of the juicy, erythematous nodule showed reticular degeneration of the epidermis with subepidermal edema and a dense band-like mixed cellular infiltrate centered in the papillary dermis with extension into the deep dermis and focally into the superficial subcutis (Fig. 2). The cellular infiltrate consisted of a small number of mature neutrophils, many immature cells that spanned the spectrum of myeloid differentiation, histiocytes and lymphocytes. A smaller proportion of the immature myeloid cells were morphologically consistent with myelocytes, with round nuclei which occupied half the cell; the majority of the immature precursors were morphologically consistent with metamyelocytes, with reniform or folded nuclei with inconspicuous nucleoli and a nuclear to cytoplasmic ratio of $1: 3$. Both the myelocytes and metamyelocytes had a moderate amount of eosinophilic cytoplasm. These precursor cells were not morphologically consistent with blasts and showed clear evidence of maturation to a small number of mature multilobated neutrophils (Fig. 3). Immunostains showed that these immature cells were positive for GD68 (KP1), lysozyme, GD45Ro (A6) and most importantly, MPO (Fig. 2); a subset of these cells was also positive for CD15 (Leu-M1). No positive staining for CD34 was observed. Scattered histiocytes were CD68-positive and lysozyme- and MPO-negative. The lymphocytes were predominantly CD3-positive T cells with only rare CD20positive $\mathrm{B}$ cells. No bacteria, fungi or acid-fast bacilli were seen in Brown-Hopps tissue gram, Gomori methenamine silver (GMS) or Fite stains, respectively. No tissue culture from the skin lesion was obtained. Scrapings of the skin lesion were submitted for direct fluorescent antibody testing for herpes simplex and varicella-zoster viruses, which were negative.

A bone marrow biopsy, that was obtained 4 days prior to the skin biopsy, showed a 40-50\% cellular marrow with all three cell lines present. A marked granulocytic predominance with left shift, because of lack of maturation to segmented neutrophils, consistent with a granulocytic maturation arrest was observed. A 300-cell differential count showed no increase in blasts $(0.3 \%)$, a relative increase in promyelocytes $(47.7 \%)$ and decrease in other granulocytic precursors and neutrophils (2.7\%). 


\section{Wu et al.}
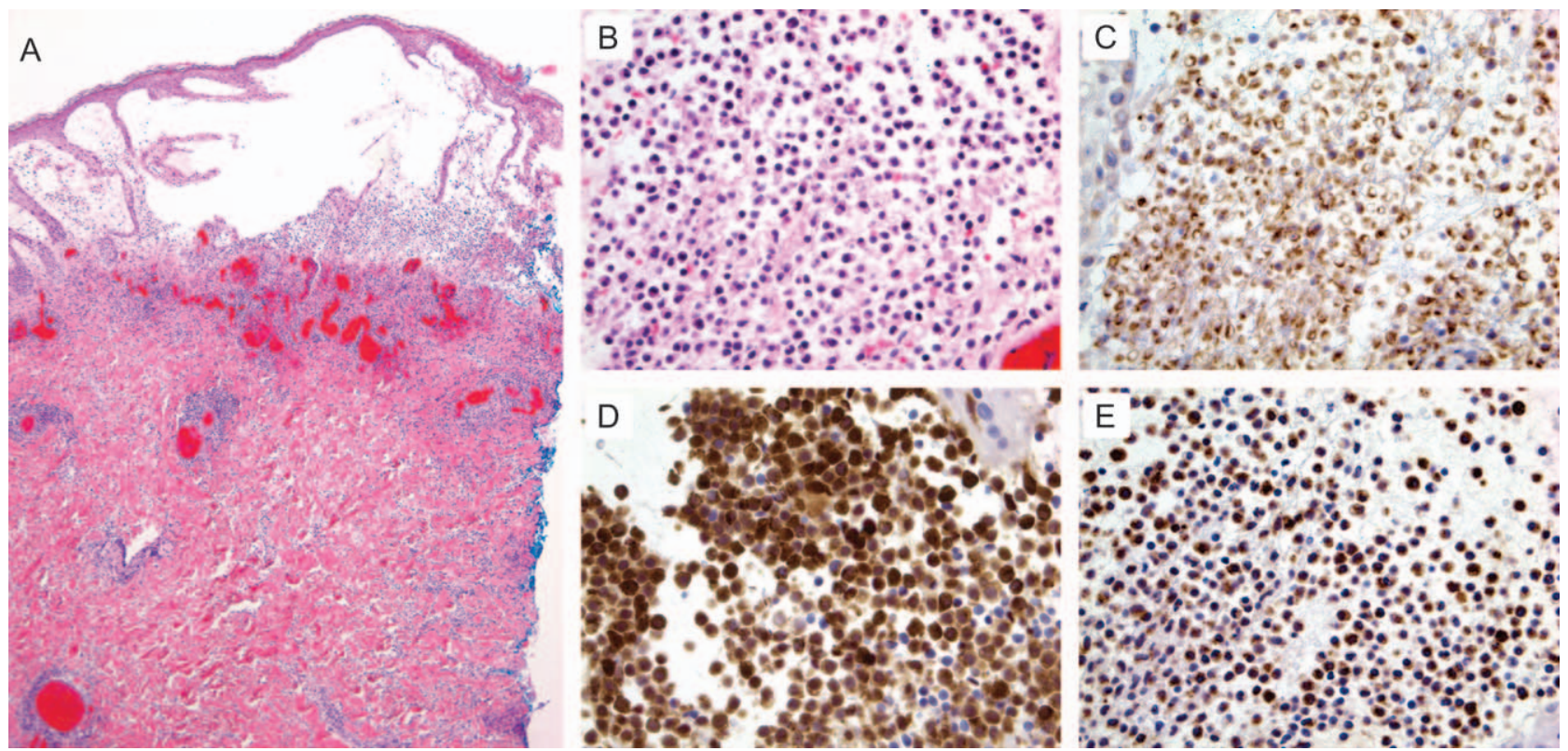

Fig. 2. Histopathologic features of the patient's skin biopsy. A) Low-power view shows a dense band-like cellular infiltrate centered in the papillary dermis with extension into the deep dermis and superficial subcutis ( $\times 4$, hematoxylin and eosin). B) The infiltrate was composed primarily of immature myeloid cells. These cells were positive for CD68, C), lysozyme D) and myeloperoxidase (MPO) E) (B-E, X40).

The promyelocytes and other granulocytic precursors were morphologically unremarkable. A cytomegalovirus viral culture of the bone marrow was negative.

Flow cytometric analysis of the bone marrow aspirate was phenotypically consistent with maturation arrest and showed no evidence of leukemia. Cytogenetic analysis of the bone marrow showed a normal female karyotype. Interphase fluorescence in situ hybridization analysis showed no evidence for the promyelocytic leukemia/retinoic acid receptor-alpha fusion associated with acute promyelocytic leukemia.

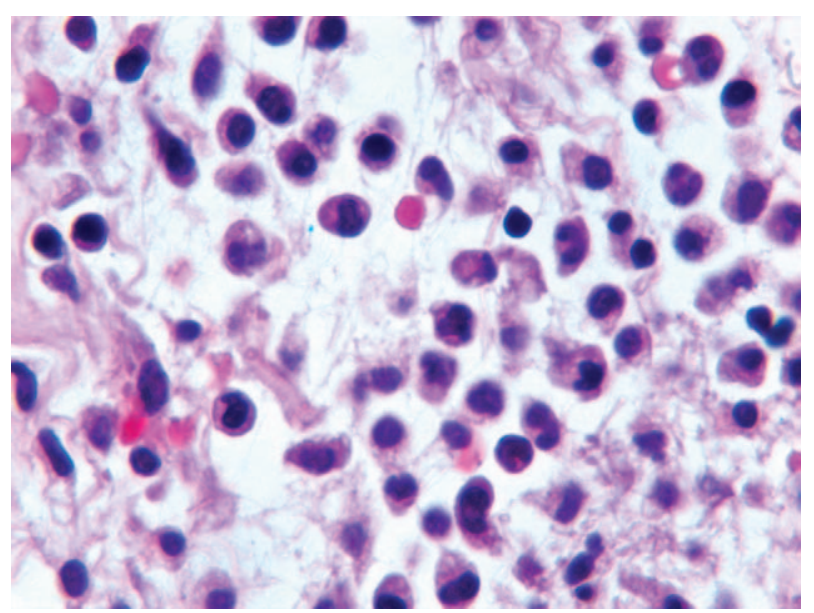

Fig. 3. The dermal cellular infiltrate was composed primarily of cells with folded nuclei, inconspicuous nucleoli and moderate eosinophilic cytoplasm; these cells were morphologically consistent with immature myeloid precursors $(\times 100$, oil immersion).

\section{Follow up}

The patient became afebrile and her WBC and ANC counts recovered with intravenous antibiotics for a presumed infection over the course of her 2-day admission (WBC 6,200 $\mathrm{mm}^{3}$ and ANC $2,500 \mathrm{~mm}^{3}$ at discharge). No topical or systemic corticosteroid therapy was given. She was discharged with a course of prophylactic Levofloxacin. At follow-up appointments, 1 week and 1 month after discharge, the erythematous nodule was noted to have spontaneously regressed.

\section{Discussion}

In the series reported by Requena et al. 41 patients with histiocytoid Sweet's syndrome were described. Associated underlying systemic diseases were present in only 11 patients, the majority of which were hematologic malignancies/disorders [chronic monocytic leukemia, lymphoma (not further specified), monoclonal gammopathy of uncertain significance, chronic B lymphocytic leukemia and multiple myeloma]. Two patients had associated solid malignancies (renal carcinoma and breast carcinoma). One patient was on immunosuppression for colitis ulcerosa. In none of these patients was histiocytoid Sweet's syndrome linked to a particular medication or to maturation arrest in the bone marrow. In this series, the authors proposed that histiocytoid Sweet's syndrome was an early stage of Sweet's syndrome. They speculated that the cellular infiltrate in these lesions would later evolve into 


\section{Drug-associated histiocytoid Sweet's syndrome}

mature, segmented neutrophils of typical Sweet's syndrome. $^{2}$

This case is unusual in that the histiocytoid Sweet's-like reaction in the skin and maturation arrest of the bone marrow occurred after TMP-SFX therapy. Although a spider bite was suspected clinically, the temporal association of this skin lesion with exposure to TMP-SFX, resolution of the skin lesion with cessation of the medication, lack of central ulceration of the lesion and failure of the patient to recall an arthropod bite at the site did not support this diagnosis. In order to definitively establish a drug-associated etiology for this process, however, it would be necessary to rechallenge the patient with the agent to see if the same response occurs; this was not undertaken as a result of the severity of the initial reaction and because this is a well described although uncommon complication of this medication. An atypical presentation of cutaneous infection was also considered; however, lack of viral inclusions and negative direct fluorescence antibody assays of epithelial cells for herpes antigen and for varicella-zoster antigen ruled out an atypical herpes virus infection, and no bacteria, fungal elements or acid-fast bacilli were identified in Brown-Hopps tissue gram, GMS and Fite stains, respectively. Extramedullary hematopoiesis was considered as well. However, the clinical presentation, which included fever, as well as the lack of a mixed cellular infiltrate including erythroid precursors and/or megakaryocytes argued against this etiology.

There are two possible pathogenic explanations for the skin lesion in this patient. First, the TMPSFX therapy may have independently caused both granulocytic maturation arrest and the skin lesion with features of histiocytoid Sweet's-like reaction. Various hematologic adverse effects because of TMP-SFX are rare but well documented and include anemia, thrombocytopenia and granulocytopenia ranging from mild to life threatening. ${ }^{3}$ The pathogenesis of non-chemotherapy drug-induced agranulocytosis is not well understood. Theories include immune/allergic mechanisms, such as the formation of anti-neutrophil antibodies and toxic mechanisms such as direct toxicity of drug metabolites against granulocytes. The idiosyncratic nature of these hematologic side effects is probably because of genetic polymorphism. ${ }^{4}$ Drug-induced Sweet's syndrome following TMP-SFX therapy has been reported but is rare. In contrast to our case, all previous reports of TMP-SFX-associated Sweet's syndrome lacked neutropenia because of a granulocytic maturation arrest. Although the pathogenesis of drug-induced Sweet's syndrome is poorly understood, it has been proposed that certain drugs may cause a rise in endogenous levels of granulocyte colony stimulating factor $(\mathrm{G}-\mathrm{CSF}){ }^{5}$
The second pathogenic explanation for the cutaneous lesion in this patient is that TMP-SFX caused an idiosyncratic granulocytic maturation arrest in the bone marrow. During the recovery phase from this acute bone marrow insult, elevated endogenous levels of G-CSF resulted in release of myeloid precursors from the bone marrow into the skin resulting in a solitary lesion of histiocytoid Sweet's-like reaction in response to an unidentified local stimulus.

G-CSF is a cytokine that is critical in granulocytic mobilization and expansion, differentiation to segmented neutrophils and neutrophilic survival. In healthy ${ }^{1}$ individuals, low levels of G-CSF maintain neutrophilic homeostasis. ${ }^{6-8}$ The level of endogenous G-CSF increases during episodes of stress or neutropenia and thus is thought to play a key role in bone marrow neutrophilic recovery. ${ }^{9}$

G-CSF, both endogenous and exogenous, has been a commonly implicated mechanism for Sweet's syndrome. Levels of endogenous G-CSF have been found to be significantly higher in patients with active Sweet's syndrome than in patients with inactive Sweet's syndrome. ${ }^{6}$ In addition, there has been several case reports of exogenous G-CSFassociated Sweet's syndrome. ${ }^{10-16}$ Notably, one case report of G-CSF-induced Sweet's syndrome described a skin biopsy in which the infiltrate had a number of immature neutrophil precursors. ${ }^{16}$ The pathogenesis of G-CSF-induced Sweet's syndrome is poorly understood, but one theory is that the G-CSF-stimulated neutrophils released during neutrophilic recovery are functionally abnormal. These neutrophils may be primed for pathologic initiation of respiratory burst activity and release of neutrophilic enzymes, leading to tissue and vessel wall damage. In addition, abnormal expression of adhesion molecules by these neutrophils may lead to endothelial adhesion and dermal chemotaxis. Alternatively, circulating secondary cytokines, such as interleukin 6 or tumor necrosis factor $\alpha$ may play a role in pathological activation of these neutrophils. ${ }^{17-19}$

In conclusion, we present a novel case of a solitary nodule presenting with a histiocytoid Sweet's-like tissue reaction pattern following granulocytic maturation arrest because of TMP-SFX. In correlating the biopsy result with the clinical appearance of the skin lesion, the dermatologist's diagnosis was a solitary, atypical Sweet's lesion. Knowledge of the association between a histiocytoid Sweet's-like reaction pattern and TMP-SFX and careful study of the cytomorphologic features of the cellular infiltrate may be important in preventing an erroneous diagnosis of myelodysplastic- or leukemia-associated Sweet's syndrome and potential overtreatment of the patients. In the future, further delineation of specific 


\section{Wu et al.}

clinical and laboratory characteristics associated with this form of Sweet's-like reaction would be helpful in clarifying the underlying pathogenetic mechanism involved in this process.

\section{References}

1. Gohen PR, Kurzrock R. Sweet's syndrome revisited: a review of disease concepts. Int J Dermatol 2003; 42: 761.

2. Requena L, Kutzner H, Palmedo G, et al. Histiocytoid sweet syndrome: a dermal infiltration of immature neutrophilic granulocytes. Arch Dermatol 2005; 141: 834.

3. Masters P, O'Bryan T, Zurlo J, Miller D, Joshi N. Trimethoprim-sulfamethoxazole revisited. Arch Intern Med 2003; 163: 402.

4. Andres E, Kurtz JE, Maloisel F. Nonchemotherapy druginduced agranulocytosis: experience of the Strasbourg teaching hospital (1985-2000) and review of the literature. Clin Lab Haematol 2002; 24: 99.

5. Walker D, Cohen P. Trimethoprim-sulfamethoxazole-associated acute febrile neutrophilic dermatosis: case report and review of drug-induced Sweet's syndrome. J Am Acad Dermatol 1996; 34: 918.

6. Kawakami T, Syuichiro O, Kawa Y, et al. Elevated serum granulocyte colony-stimulating factor levels in patients with active phase of Sweet syndrome and patients with active Behcet disease. Arch Dermatol 2004; 140: 570.

7. Avalos B, Gasson J, Hedvat C, et al. Human granulocyte colony-stimulating factor: biologic activities and receptor characterization on hematopoietic cells and small cell lung cancer cell lines. Blood 1990; 75: 851.

8. Philpott NJ, Prue RL, Marsh JC, Gordon-Smith EC, Gibson FM. G-CSF-mobilized CD34+ peripheral blood stem cells are significantly less apoptotic than unstimulated peripheral blood CD34+ cells: role of G-CSF as survival factor. Br J Haematol 1997; 97: 146.
9. Kavgaci H, Ozdemir F, Aydin F, Yavuz A, Yavuz M. Endogenous granulocyte colony-stimulating factor (G-CSF) levels in chemotherapy-induced neutropenia and in neutropenia related with primary diseases. J Exp Clin Cancer Res 2002; 21: 475 .

10. Arbetter KR, Hubbard KW, Markovic SN, Gibson LE, Phyliky RL. Case of granulocyte colony-stimulating factor-induced Sweet's syndrome. Am J Hematol 1999; 61: 126.

11. Fukutoku M, Shimizu S, Ogawa Y, et al. Sweet's syndrome during therapy with granulocyte colony-stimulating factor in a patient with aplastic anaemia. Br J Haematol 1994; 86: 645.

12. Hasegawa M, Sato S, Nakada M, et al. Sweet's syndrome associated with granulocyte colony-stimulating factor. Eur J Dermatol 1998; 8: 503.

13. Prevost-Blank PL, Shwayder TA. Sweet's syndrome secondary to granulocyte colony-stimulating factor. J Am Acad Dermatol 1996; 35: 995.

14. Richard M, Grob J, Laurans R, et al. Sweet's syndrome induced by granulocyte colony-stimulating factor in a woman with congenital neutropenia. J Am Acad Dermatol 1996; 35: 629 .

15. White J, Mufti G, Salisbury J, du Vivier A. Cutaneous manifestations of granulocyte colony-stimulating factor. Clin Exp Dermatol 2006; 31: 206.

16. Magro CM, De Moraes E, Burns F. Sweet's syndrome in the setting of CD34-positive acute myelogenous leukemia treated with granulocyte colony stimulating factor: evidence for a clonal neutrophilic dermatosis. J Cutan Pathol 2001; 28: 90.

17. Dereure O, Hillaire-Buys D, Guilhou J. Neutrophil-dependent cutaneous side-effects of leucocyte colony-stimulating factors: manifestations of a neutrophil recovery syndrome? $\mathrm{Br} \mathrm{J}$ Dermatol 2004; 150: 1228.

18. Johnston B, Kubes P. The $\alpha$ 4integrin: an alternative pathway for neutrophil recruitment? Immunol Today 1999; 20: 545.

19. Spiekermann K, Roesler J, Emmendoerffer A, Elsner J, Welte K. Functional features of neutrophils induced by G-CSF and GM-CSF treatment: differential effects and clinical implications. Leukemia 1997; 11: 466. 